The likelihood of presenting with a PSAV $>2.0 \mathrm{ng} / \mathrm{ml}$ per year was significantly greater among men with high total PSA levels $(P<0.0001): 31 \%$ of men with a PSA levels $4.1-$ $10.0 \mathrm{ng} / \mathrm{ml}$ and $74 \%$ of men with PSA levels $>10.0 \mathrm{ng} / \mathrm{ml}$ had a PSAV > $2 \mathrm{ng} / \mathrm{ml}$ per year. In previous studies men with a PSAV of $>2 \mathrm{ng} / \mathrm{ml}$ per year were more likely to have disease progression or die following treatment than men with lower values. Those findings and the results from this study imply that a raised initial PSA is associated with poor treatment outcomes.

The authors conclude this strong association between PSA and PSAV should be considered in future studies and the development of risk assessment tools.

Original article Yu X et al. (2007) The association between total prostate specific antigen concentration and prostate specific antigen velocity. J Urol 177: 1298-1302

\section{Effects of complications on outcomes after nephrectomy for renal cancer}

Renal malignancy is treated by total nephrectomy (TN) or, if the renal mass is $\leq 4 \mathrm{~cm}$, partial nephrectomy (PN) to preserve renal function. Population-based findings on the frequency of complications and predictors of morbidity associated with both procedures are lacking; therefore, Joudi et al. set about answering these questions in a large US population-based study.

Retrospective analysis of the US Nationwide Inpatient Sample (NIS) data set of the Healthcare Cost and Utilization project identified patients who had undergone PN $(n=3,019)$ or TN $(n=18,575)$ for renal cancer between 2000 and 2003. The risk of complications following $\mathrm{PN}$ and $\mathrm{TN}$ and their effects on mortality and length of hospital stay were assessed by multivariate analysis. The roles of hospital-related factors (volume, location, teaching status, bed size) and patient-related factors (age, sex, comorbid severity index) were also investigated. PN and TN procedures were associated with similar mortality $(0.66 \%$ and $1.06 \%)$ and complication rates (17.16\% and $18.20 \%)$. The most frequent complications for both procedures were respiratory, digestive or bleedingrelated. Length of hospital stay and associated hospital charges were similar for PN and TN. Significant predictors for complications following TN were age, sex, comorbidity index and hospital location (rural versus urban), whereas comorbidity index $>2$ was the only significant predictor for complications following PN.

This population-based study reports similarly low mortality and morbidity for TN and PN, in agreement with the findings of single-institution studies.

Original article Joudi FN et al. (2007) Analysis of complications following partial and total nephrectomy for renal cancer in a population based sample. J Urol 177: 1709-1714

\section{Long-term warfarin use could reduce the risk of prostate cancer}

Secondary analyses in studies of warfarin treatment for venous thromboembolism suggest that warfarin use is associated with a reduced risk of cancers, particularly urogenital cancers; however, some reports have failed to find such an association. Tagalakis et al., therefore, carried out a population-based, nested case-control study in Saskatchewan, Canada, to assess whether warfarin use is associated with a decreased risk of urogenital cancer.

The source population (aged $\geq 50$ years with no history of cancer since 1967) was derived from beneficiaries of Saskatchewan Health who were eligible for prescription drug benefits between 1 January 1981 and 31 December 2002. During this period 19,412 cases of urogenital cancer (11,502 prostate, 3,424 bladder, 1,601 kidney, 1,800 uterine and 1,085 ovarian) were diagnosed in the source population. A total of 116,470 controls matched for age and sex and free from cancer (six for each case) were randomly selected from the source population. After adjustment for potentially confounding effects of prescription drugs, no significant association was found between warfarin use and the risk of any of these urogenital cancers apart from prostate cancer. Compared with men who never used warfarin, long-term cumulative warfarin use ( $\geq 1$ prescription per year in the 4 years immediately before cancer diagnosis) significantly decreased the adjusted incidence rate ratio of prostate cancer (0.80, 95\% Cl 0.65-0.99).

The authors conclude that the anticancer effect of long-term warfarin use for prostate cancer should be investigated further to comprehensively assess the effects of confounders on this association.

Original article Tagalakis V et al. (2007) Use of warfarin and risk of urogenital cancer: a population-based, nested casecontrol study. Lancet Oncol 8: 395-402 